\title{
ON THE SOLUTION OF A DIFFERENTIAL EQUATION WITH NONLINEARITY APPEARING IN THE SECOND DERIVATIVE OF COMBINED LINEAR AND CUBIC TERMS*
}

\author{
BY \\ CHI-NENG SHEN \\ Thayer School of Engineering, Dartmouth College
}

1. Introduction. One commonly analyzed application of automatic controls to processes is shown schematically in Fig. 1. Here a linear feedback controller is used to control a two-capacity process. The process involves two linear capacities or tanks in this case, and two linear resistances. The analysis of this process is treated in detail in the literature and in the texts on automatic control. The solution is simple and straight-

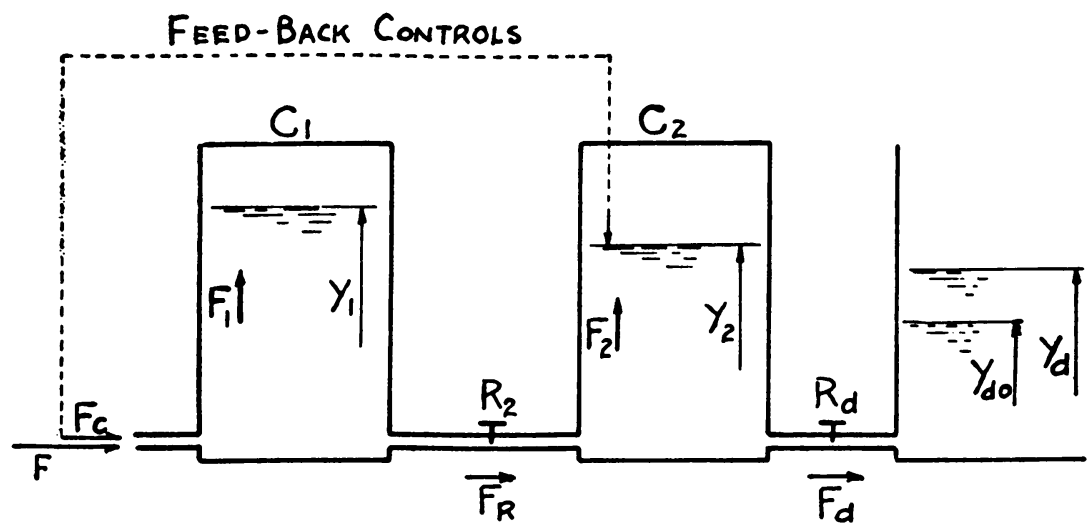

Fra. 1. Linear two-capacity process with controls.

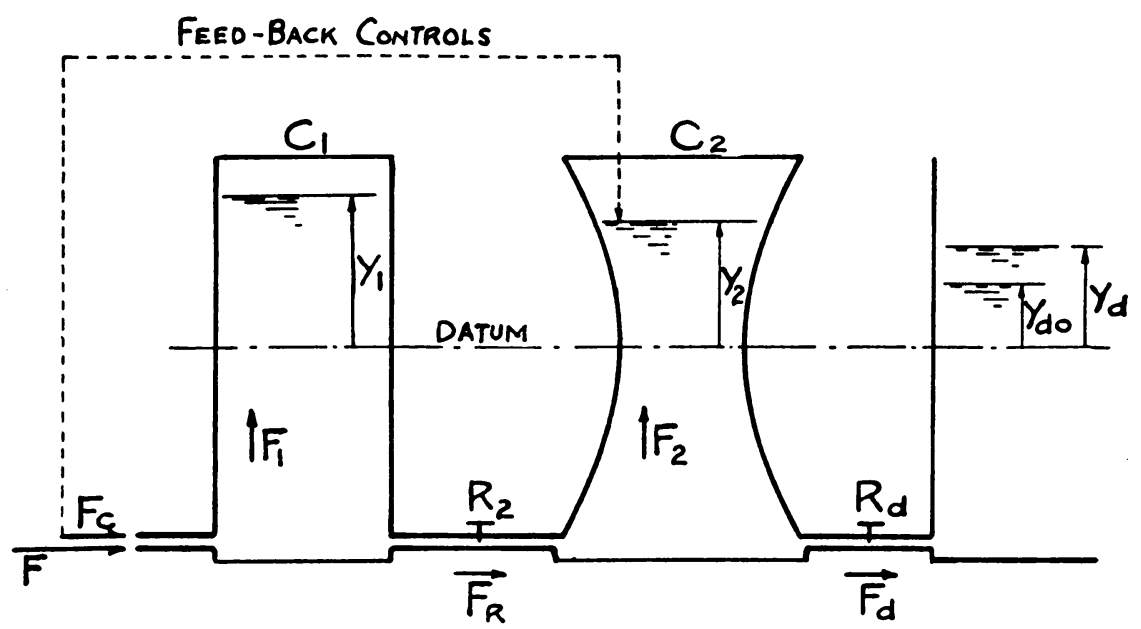

Fra. 2. Single nonlinear capacity of two-capacity process.

*Received September 13, 1955; revised manuscript received January 11, 1956. 
forward. If, however, nonlinear elements are introduced, the problem becomes more complex and cannot be solved in the conventional manner.

If tanks of the form shown in Fig. 2 are used, the capacity is nonlinear. The capacity of a parabolic tank may be expressed by an equation of the form:

$$
\text { Volume }=C\left(x+\alpha x^{3}+\gamma x^{5}\right) .
$$

If $\gamma$ is small, this equation reduces to:

$$
\text { Volume }=C\left(x+\alpha x^{3}\right) .
$$

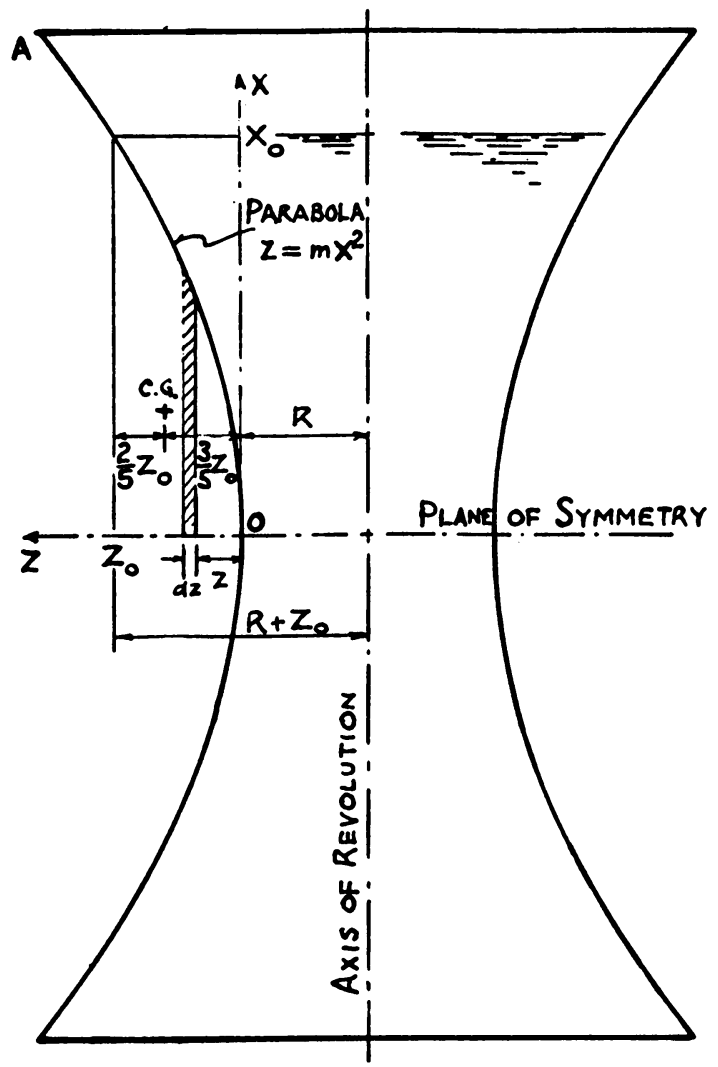

Fia. 3. Nonlinear capacity, neck shaped vessel.

In tanks of the form shown in Fig. 3, $\alpha$ is positive, and for the form shown in Fig. 4, $\alpha$ is negative. This expression also will give the approximate volume of many other tank forms.

If a system consists of tanks, the volume of which may be expressed in the form of Eq. (2), the differential equation which defines the behavior of the system may be written as:

$$
C_{1} \frac{d y_{1}}{d t}=a_{1} y_{1}+a_{2} y_{2}+a_{3} \text {, }
$$




$$
C_{2} \frac{d}{d t}\left(y_{2}+\alpha y_{2}^{8}\right)=b_{1} y_{1}+b_{2} y_{2}+b_{3} \text {, }
$$

where $a, b, c$ with subscripts are all parameters.

The purpose of this paper is to indicate a solution which describes the behavior of such a system. However to facilitate the analysis, it will be convenient to make certain transformations and to discuss the problem in terms of trajectories in the phase-plane.

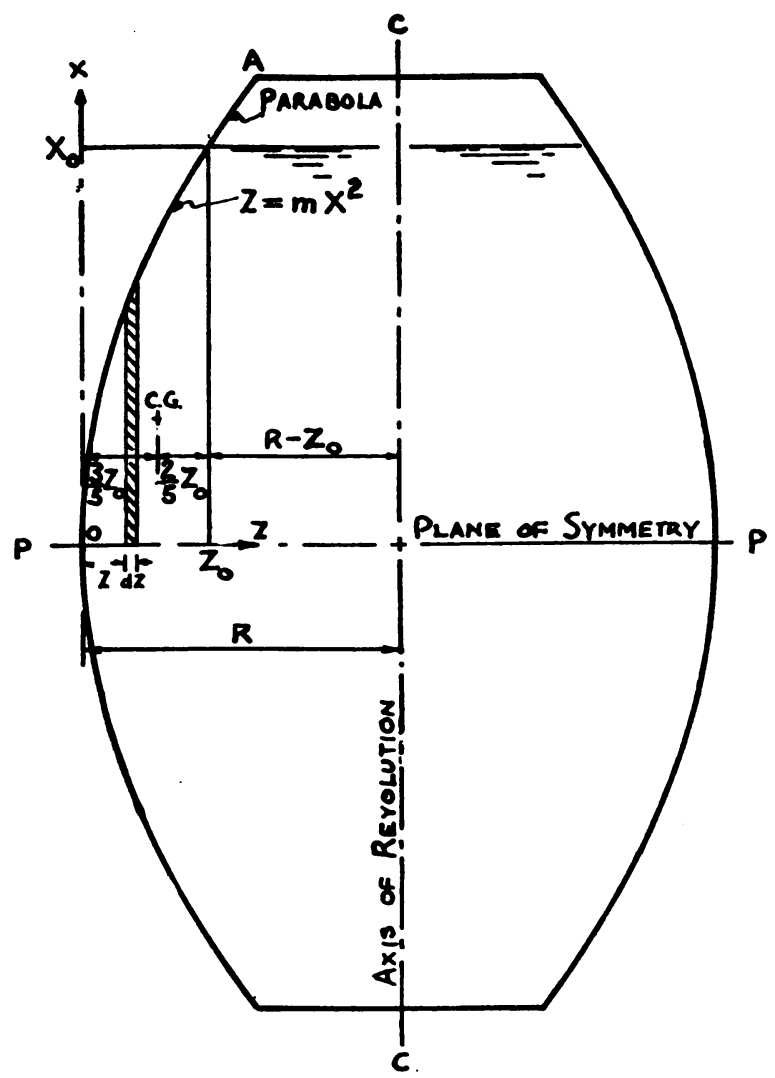

FrG. 4. Nonlinear capacity, barrel shaped vessel.

By solving for $y_{1}$ in Eq. (3b), differentiating and substituting the value of $y_{1}$ and $d y_{1} / d t$ into Eq. (3a), it can be shown that:

$$
m_{1} \frac{d^{2}}{d t^{2}}\left(y_{2}+\alpha y_{2}^{2}\right)+m_{2} \frac{d}{d t}\left(y_{2}+\alpha y_{2}^{3}\right)+m_{3} \frac{d}{d t} y_{2}+m_{4} y_{2}+m_{5}=0
$$

where $m$ 's are all parameters.

By a suitable transformation, the differential equation, Eq. (4), may be reduced to the form:

$$
\frac{d^{2}}{d \sigma^{2}}\left(Y+\beta Y^{3}\right)+M_{n} \frac{d}{d \sigma}\left(Y+\beta Y^{3}\right)+M_{1} \frac{d Y}{d \sigma}+Y=r .
$$

The quantities $\beta, M_{n}, M_{1}$, and $r$ are all parameters. This equation differs from the standard nonlinear differential equations for which solutions are available. 
If $V$ is defined as:

$$
V=\frac{d Y}{d \sigma}
$$

then

$$
\begin{aligned}
\frac{d}{d \sigma}\left(Y+\beta Y^{3}\right) & =\left(1+3 \beta Y^{2}\right) V \\
\frac{d^{2}}{d \sigma^{2}}\left(Y+\beta Y^{3}\right) & =V \frac{d}{d Y}\left[\left(1+3 \beta Y^{2}\right) V\right] .
\end{aligned}
$$

Thus, from Eq. (5)

$$
\frac{d V}{d Y}=-\left(M_{n}+\frac{M_{1}}{1+3 \beta Y^{2}}\right)+\frac{r-Y\left(1+6 \beta V^{2}\right)}{V\left(1+3 \beta Y^{2}\right)},
$$

where $V$ and $Y$ are the phase-plane variables.

A discussion of the solutions of the key equation (6) in its general form will be temporarily postponed, and a special case where $M_{n}=M_{1}=0$ will be considered, for in this case a solution in closed form can be obtained.

2. An explicit solution for the special case, $M_{n}=M_{1}=0$.

When $M_{n}=M_{1}=0$, note that Eq. (6) takes the form,

$$
\frac{V d V}{1+6 \beta V^{2}}=\frac{r d Y}{\left(1+3 \beta Y^{2}\right)\left(1+6 \beta V^{2}\right)}-\frac{Y d Y}{1+3 \beta Y^{2}} .
$$

Equation (7) does not appear to be integrable because the first term on the righthand side of the equation is a function of both $V$ and $Y$. However, by introducing a new function $\phi$ and rearranging the equation, the explicit solution of Eq. (7) may be found.

Define

$$
\phi(Y)=12 \beta r \int_{Y_{\bullet}}^{Y} \frac{d Y}{\left(1+3 \beta Y^{2}\right)\left(1+6 \beta V^{2}\right)} .
$$

Also, from Eq. (7),

$$
\phi(Y)=\int_{V_{0}}^{V} \frac{12 V d V}{1+6 \beta V^{2}}+\int_{Y_{0}}^{Y} \frac{12 \beta Y d Y}{1+3 \beta Y^{2}},
$$

where $Y_{0}$ and $V_{0}$ are the initial values of $Y$ and $V$ respectively. Equation (9) is readily integrable and

$$
\phi(Y)=\ln \frac{1+6 \beta V^{2}}{1+6 \beta V_{0}^{2}} \frac{\left(1+3 \beta Y^{2}\right)^{2}}{\left(1+3 \beta Y_{0}^{2}\right)^{2}} .
$$

Let $\phi_{0}$ be the initial value of $\phi$. Using Eq. (10) the value of $\phi_{0}$ may be determined; in fact

$$
\phi(Y)_{\substack{\operatorname{at}\left(Y-Y_{0}\right) \\\left(V-V_{0}\right)}}=\phi_{0}=\ln 1=0
$$

thus

$$
\epsilon^{+}=\frac{\left(1+6 \beta V^{2}\right)\left(1+3 \beta Y^{2}\right)^{2}}{Q^{2}},
$$


where

$$
Q^{2}=\left(1+6 \beta V_{0}^{2}\right)\left(1+3 \beta Y_{0}^{2}\right)^{2} .
$$

Combining Eq. (8) and Eq. (11), the variable $V$ may be eliminated and

$$
\phi(Y)=12 \beta r \int_{Y_{0}}^{Y} \frac{\epsilon^{-\phi}\left(1+3 \beta Y^{2}\right) d Y}{Q^{2}} .
$$

Differentiating Eq. (12),

$$
\frac{d \phi}{d Y}=12 \beta r \frac{\epsilon^{-\phi}\left(1+3 \beta Y^{2}\right)}{Q^{2}} .
$$

Now, the variables are separable and thus by integration

$$
\left.\left.\epsilon^{*}\right]_{\phi_{0}}^{\phi}=\frac{12 \beta r}{Q^{2}}\left(Y+\beta Y^{3}\right)\right]_{Y_{0}}^{Y}
$$

or

$$
\epsilon^{\phi}=1+\frac{12 \beta r}{Q^{2}}\left[\left(Y+\beta Y^{3}\right)-\left(Y_{0}+\beta Y_{0}^{3}\right)\right] .
$$

Combining Eqs. (11) and (14), the solution of Eq. (7) may be obtained:

$$
\left(1+6 \beta V^{2}\right)\left(1+3 \beta Y^{2}\right)^{2}=Q^{2}+12 \beta r\left[\left(Y+\beta Y^{3}\right)-\left(Y_{0}+\beta Y_{0}^{3}\right)\right]
$$

or

$$
V^{2}=\frac{S^{2}-\left(Y^{2}+1.5 \beta Y^{4}\right)+2 r\left(Y+\beta Y^{3}\right)}{\left(1+3 \beta Y^{2}\right)^{2}}
$$

where

$$
S^{2}=Y_{0}^{2}+1.5 \beta Y_{0}^{4}+V_{0}^{2}\left(1+3 \beta Y_{0}^{2}\right)^{2}-2 r\left(Y_{0}+\beta Y_{0}^{3}\right)
$$

is a function of the starting conditions. Equation (15) together with Eq. (16) yields the solution of the differential Eq. (7) in closed form.

3. Bounded and unbounded solutions for $r=0$ when $M_{n}=M_{1}=0$. Bounded and unbounded solutions in the special case under consideration will be investigated. For any real value of $Y$ and any positive value of $\beta$ it may be seen that $\left(1+3 \beta Y^{2}\right)^{2}>1$ and from Eq. (11), setting $r=0$, that

$$
V^{2}<S^{2}-\left(Y^{2}+1.5 \beta Y^{4}\right),
$$

or

$$
V^{2}+Y^{2}+1.5 \beta Y^{4}<S^{2}
$$

where

$$
S^{2}=Y_{0}^{2}+1.5 \beta Y_{0}^{4}+V_{0}^{2}\left(1+3 \beta Y_{0}^{2}\right)^{2} .
$$

Thus $V$ and $Y$ are bounded in the phase plane as shown in Eq. (17) where the lefthand quantity has to be smaller than a positive real number $S^{2}$.

However, for a negative value of $\beta$ the situation may be quite different. A plot of the solution of Eq. (7) on the phase-plane from Eq. (15) and Eq. (16) will demonstrate 
this. It is clear that the solution of Eq. (7) depends on the starting conditions in the phase-plane. A numerical example where $\beta$ is negative will illustrate this feature.

Assume

$$
\beta=-\frac{4}{300}, \quad r=0,
$$

then

$$
V^{2}=\frac{S^{2}-Y^{2}+.02 Y^{4}}{\left(1-.04 Y^{2}\right)^{2}}
$$

These phase plane trajectories are plotted in Fig. 5. At $S^{2}=12.5$, the value of $|V|$ is 3.535 for every value of $Y$, except $|Y|=5.00$. At $|Y|=5.00, V$ is indeterminate. The system is bounded provided the initial point $\left(Y_{0}, V_{0}\right)$ falls within the rectangular box $|Y| \leq 5,|V| \leq 3.535$. An initial point falling outside the box gives rise to an unbounded trajectory.

For example, if the starting conditions are given as

$$
Y_{0}=+4, \quad V_{0}=+4
$$

we can see that the system is not bounded.

4. The effect of changing the parameter $\beta$. The choice of any particular value for $\beta$, for example $\beta= \pm 4 / 300$, in no way limits the generality of the treatment, for the characteristic of the solution for any other value of $\beta$ can be obtained by introducing a scale factor, i.e., a transformation parameter may be used to make the coefficient $\beta$ equal to $\pm 4 / 300$. More precisely, if this parameter is denoted by $L$, let $Y=L Z$, then Eq. (5) may be written as

$$
\frac{d^{2}}{d \sigma^{2}}\left(Z+\beta L^{2} Z^{3}\right)+M_{n} \frac{d}{d \sigma}\left(Z+\beta L^{2} Z^{3}\right)+M_{1} \frac{d Z}{d \sigma}+Z=\frac{r}{L},
$$

or

$$
\frac{d^{2}}{d \sigma^{2}}\left(Z+\xi Z^{3}\right)+M_{n} \frac{d}{d \sigma}\left(Z+\xi Z^{3}\right)+M_{1} \frac{d Z}{d \sigma}+Z=R,
$$

where

$$
\xi=\beta L^{2} \text { and } R=\frac{r}{L} .
$$

Hence $\xi$ may be made equal to either $+4 / 300$ or $-4 / 300$ by a suitable scale factor $L^{2}$ and $R$ can be calculated correspondingly. The solution of Eq. (5) will be $Y=L Z$, where $Z$ is readily obtainable by comparing Eq. (18) with Eq. (5). These remarks apply not only to the general equation, but also to the special equation arising when $M_{n}=$ $M_{1}=0$.

5. Bounded and unbounded solutions for $r \neq 0$ when $M_{n}=M_{1}=0$. In Sec. 3 it was shown that a solution would be bounded when $r=0$ if the initial values $\left(Y_{0}, V_{0}\right)$ fell within a certain rectangle (see Fig. 5). When $r \neq 0$ this bounded region changes shape. Indeed the bounded region for Eq. (7) with a negative nonlinearity has a horseshoe shape. 


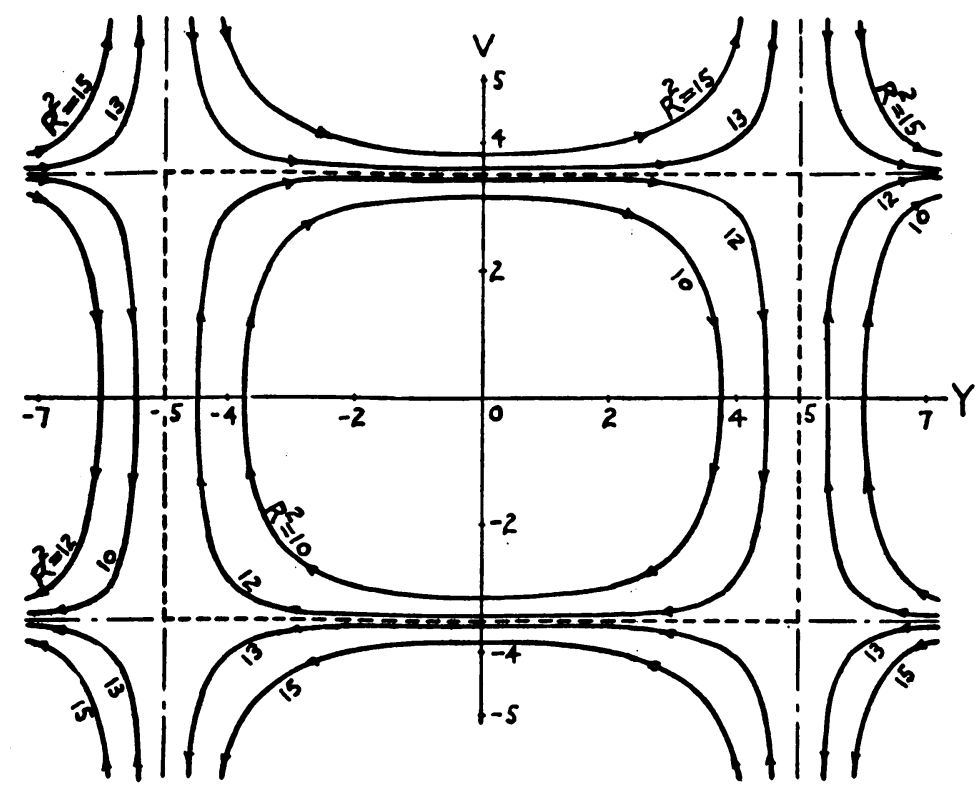

Fic. 5. Negative nonlinearity for $\beta=-4 / 300$ and $r=0$.

To demonstrate this, Eq. (15) may be re-examined, keeping in mind that a bounded value of $V$ is required as $1+3 \beta Y^{2}$ approaches zero. In this case the numerator of the righthand side of Eq. (15) also approaches zero

$$
S^{2}-\left(Y^{2}+1.5 \beta Y^{4}\right)+2 r\left(Y+\beta Y^{3}\right) \rightarrow 0 .
$$

This is the criterion for a boundary of the bounded region.

By substituting $Y^{2}=-1 / 3 \beta-\epsilon$ into Eq. (20), we have

$$
S^{2}=-\frac{1}{6 \beta}-\frac{4 r}{3}\left(-\frac{1}{3 \beta}\right)^{1 / 2}+\epsilon_{1},
$$

where $\epsilon$ and $\epsilon_{1}$ are all infinitesimals.

Since $\beta$ is negative, $\beta^{\prime}$ may be defined as $\beta^{\prime} \equiv-\beta$. Using this value, $\beta^{\prime}$, and the value of $S^{2}$ from Eq. (16), Eq. (21) becomes

$$
V_{0}^{2}=\frac{\left(1 / 6 \beta^{\prime}\right)-(4 r / 3)\left(1 / 3 \beta^{\prime}\right)^{1 / 2}+2 r\left(Y_{0}-\beta^{\prime} Y_{0}^{3}\right)-Y_{0}^{2}+1.5 \beta^{\prime} Y_{0}^{4}}{\left(1-3 \beta^{\prime} Y_{0}^{2}\right)^{2}}
$$

The above equation determines the bounded region for Eq. (7).

It is interesting to note that if $Y_{0}^{2}=1 / 3 \beta^{\prime}, V_{0}^{2}$ is indeterminate. However, the value of $V_{0}^{2}$ can be found as $Y_{0}^{2} \rightarrow 1 / 3 \beta^{\prime}$ by differentiating the numerator and denominator of Eq. (22) with respect to $Y_{0}$; in fact

$$
\lim _{Y_{0} \rightarrow\left(1 / 3 \beta^{\prime}\right) 2 /:} V_{0}^{2}=\frac{2 r-6 \beta^{\prime} r Y_{0}^{2}-2 Y_{0}+6 \beta^{\prime} Y_{0}^{3}}{2\left(1-3 \beta^{\prime} Y_{0}^{2}\right)\left(-6 \beta^{\prime} Y_{0}\right)}=\frac{Y_{0}-r}{6 \beta^{\prime} Y_{0}}
$$


If the solutions to Eqs. (22) and (23) are plotted for $r=0.1,1.0,2.0,4.0$ at $\beta=$ $-\beta^{\prime}=-4 / 300$, the boundary is seen to be of $U$ or horseshoe shape as shown in Fig. 6 .

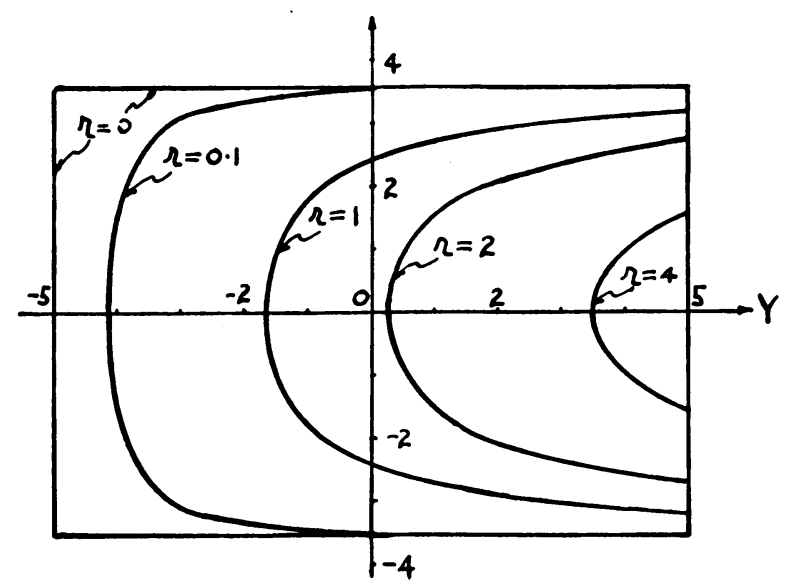

Fra. 6. Bounded regions for different $r, \beta=-4 / 300$.

At a particular value of $r$, the solution is bounded when any set of starting conditions lay within the horseshoe region; otherwise it is unbounded. The phase plane diagram for Eq. (15) is also given for $r=1.0$; see Figs. 7 and 8.

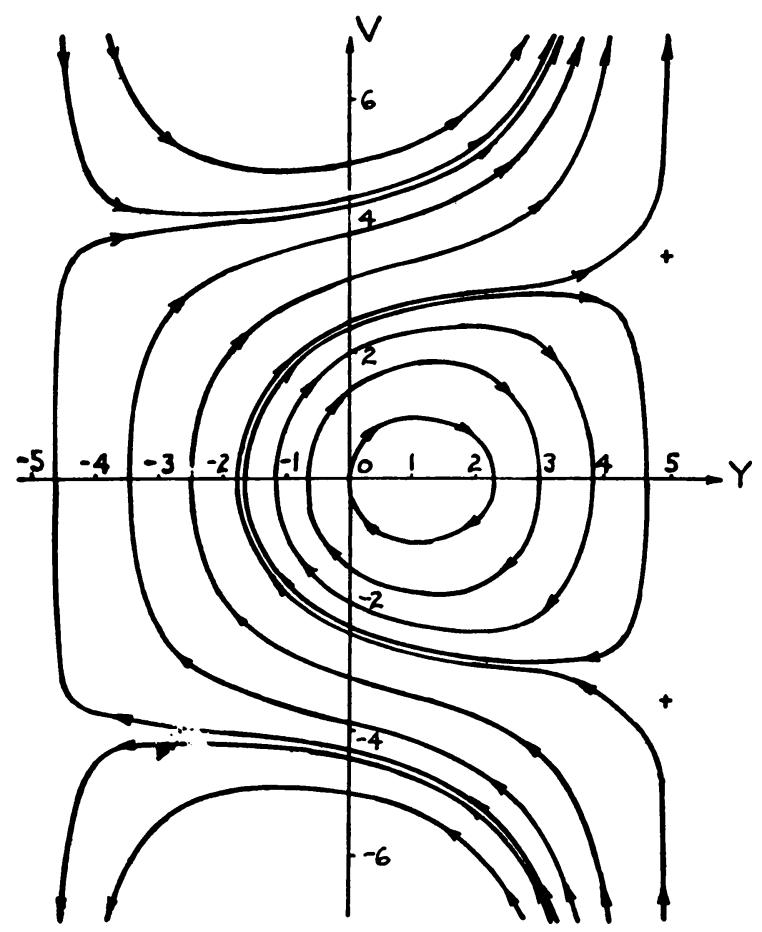

Fra. 7. Negative nonlinearity for $\beta=-4 / 300$ and $r=1.0$. 


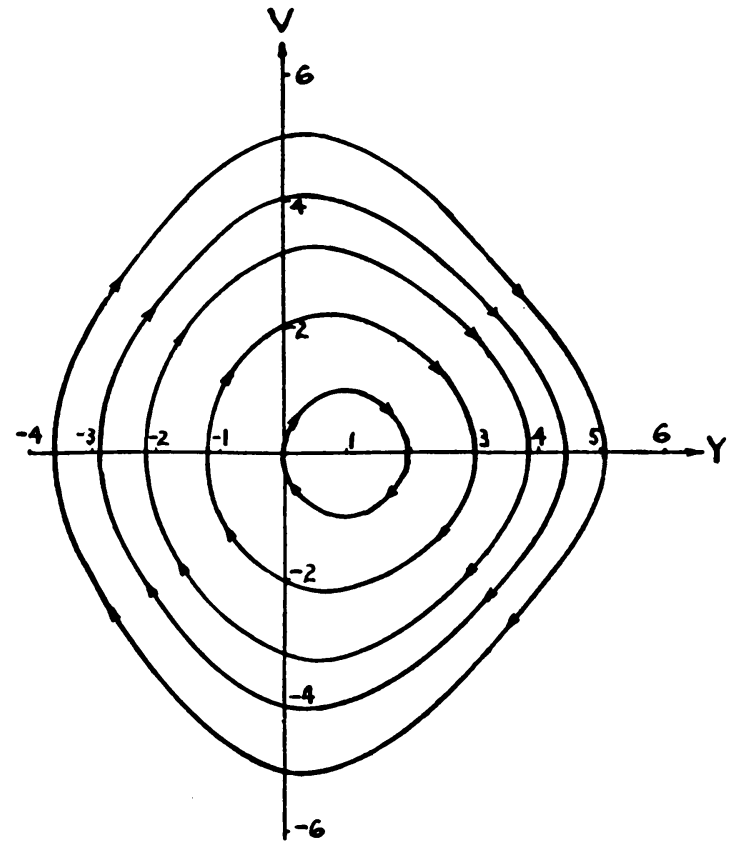

Fra. 8. Positive nonlinearity for $\beta=+4 / 300$ and $r=1.0$.

6. The period and frequency when $M_{n}=M_{1}=0$. The period of a bounded solution of Eq. (7) is in general an elliptic integral. This can be seen by rearranging Eq. (15) in the form

$$
\int_{0}^{\sigma} d \sigma=\int_{0}^{y} \frac{\left(1+3 \beta Y^{2}\right) d Y}{\left[S^{2}-\left(Y^{2}+1.5 \beta Y^{4}\right)+2 r\left(Y+\beta Y^{3}\right)\right]^{1 / 2}} .
$$

If numerical values are assigned to the parameters, the integral of Eq. (24) may be evaluated by referring to any standard text on elliptic integrals.

If $r=0$, the general solution can be found by considering

$$
\frac{d^{2}}{d \sigma^{2}}\left(Y+\beta Y^{3}\right)+Y=0 .
$$

Expressing $\sigma$ in terms of $Y$, Eq. (25) may be written in the form

$$
\int_{0}^{D} d \sigma=\int_{0}^{Y_{m}} \frac{\left(1+3 \beta Y^{2}\right) d Y}{\left[S^{2}-Y^{2}-1.5 \beta Y^{4}\right]^{1 / 2}},
$$

where

$$
S^{2}=Y_{0}^{2}+1.5 \beta Y_{0}^{4}+V_{0}^{2}\left(1+3 \beta Y_{0}^{2}\right)^{2} .
$$

The solution of Eq. (26) is a combined elliptic integral of the first and second kind. If $\beta$ is negative, then the quarter period will be

$$
p=\left(\frac{Y_{m}}{S}-\frac{2 S}{Y_{m}}\right) K(k)+\frac{2 S}{Y_{m}} E(k),
$$


where

$$
Y_{m}^{2}=\frac{1-\left[1-6 \beta^{\prime} S^{2}\right]^{1 / 2}}{3 \beta^{\prime}}, \quad k=\left[\frac{Y_{m}^{2}-S^{2}}{S^{2}}\right]^{1 / 2},
$$

$Y_{m}$ being the maximum value of $Y$ at $V=0$, if bounded and $\beta^{\prime} \equiv-\beta . K$ denotes the complete elliptical integral of the first kind. $E$ denotes the complete elliptical integral of the second kind. The general solution of Eq. (26) will be in the form

$$
\dot{\sigma}=\left(\frac{Y_{m}}{S}-\frac{2 S}{Y_{m}}\right) F(\theta, k)+\frac{2 S}{Y_{m}} E(\theta, k),
$$

where

$$
\theta=\sin ^{-1} \frac{Y}{Y_{m}}
$$

If $\beta$ is positive, the quarter period will be

$$
p=+\left[2\left(\frac{S}{Y_{m}}\right)^{2}-1\right]^{1 / 2}[2 E(k)-K(k)]
$$

where

$$
Y_{m}^{2}=\frac{-1+\left[1+6 \beta S^{2}\right]^{1 / 2}}{3 \beta}, \quad k=\left[\frac{S^{2}-Y_{m}^{2}}{2 S^{2}-Y_{m}^{2}}\right]^{1 / 2},
$$

$Y_{m}$ being the maximum value of $Y$ at $V=0$. The general solution of Eq. (26) will be in the form

$$
\sigma=\left[2\left(\frac{S}{Y_{m}}\right)^{2}-1\right]^{1 / 2}\left[2 E\left(\frac{\pi}{2}, k\right)-2 E(\theta, k)-K\left(\frac{\pi}{2}, k\right)+F(\theta, k)\right],
$$

where

$$
\theta=\cos ^{-1} \frac{Y}{Y_{m}}
$$

The frequency may be calculated for both negative and positive $\beta$ as

$$
\omega=\frac{\pi / 2}{p} \text {. }
$$

The frequencies for various values of $Y_{m}$ and for $\beta= \pm 4 / 300$ are plotted in Fig. 9 . It is interesting to compare Eq. (25) with the Duffing's equation,

$$
\frac{d^{2}}{d \tau^{2}} X+X+\lambda X^{3}=0
$$

where the period is an elliptic integral of the first kind only. If $\beta$ and $\lambda$ are small, the approximation $\lambda=-\beta=\beta^{\prime}$ may be used.

Therefore the negative nonlinearity of Eq. (25) corresponds to the positive nonlinearity of the Duffing's equation, and vice versa. This can be seen as the positive branch curves towards the lower frequencies just as the negative branch does for the Duffing's equation. However, the frequency will be entirely different as starting amplitude gets higher. 


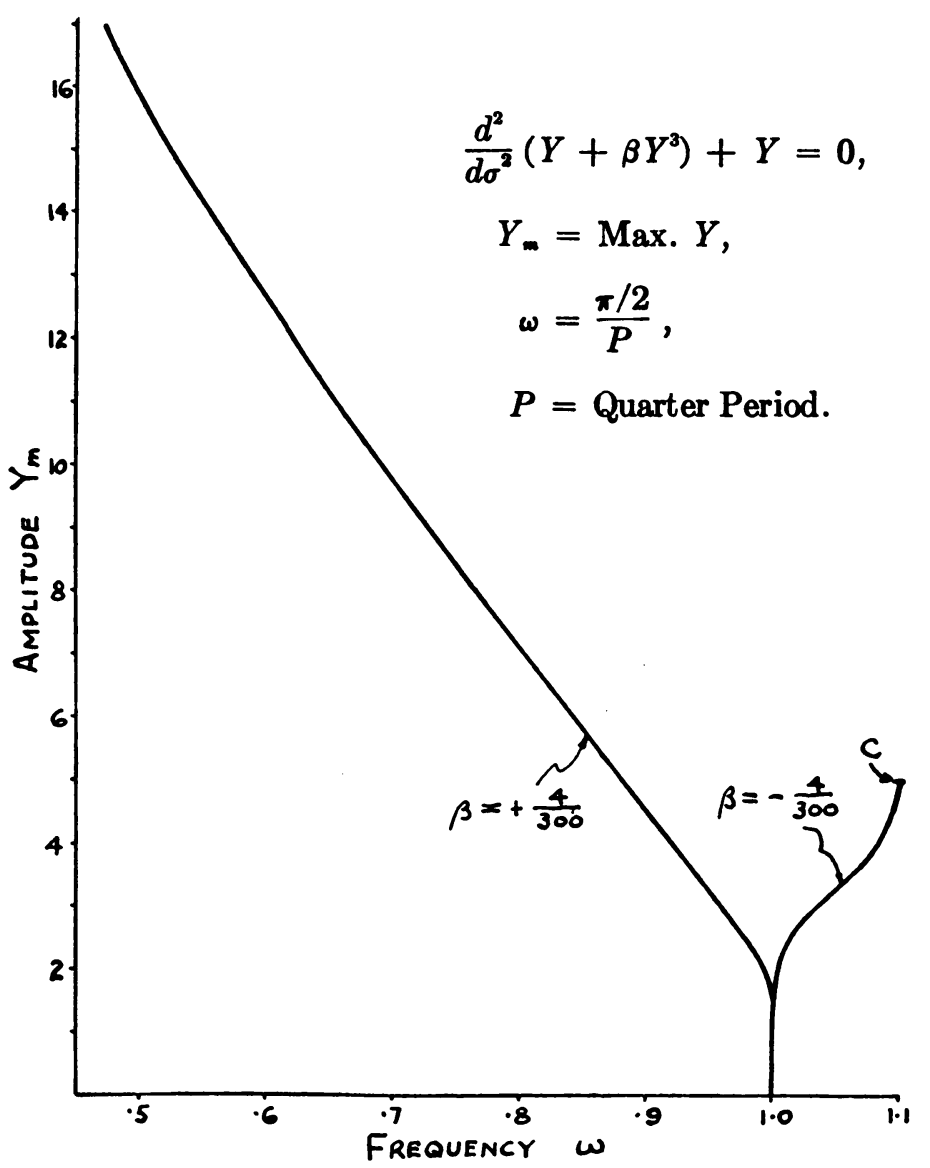

Fra. 9. Frequency amplitude response. $c$ denotes cut-off point at $Y_{m}=5$.0.

For a very high amplitude the frequency slows down for a positive $\beta$, while there is no bounded solution for a negative $\lambda$ with a very large amplitude in the Duffing's equation. For a negative $\beta$, the amplitude is restricted and not more than $\left(1 / 3 \beta^{\prime}\right)^{1 / 2}$, while there is always a frequency for any positive $\lambda$ at high amplitude for Eq. (35).

7. Solution with damping terms. With all these details in mind for the characteristic of solutions of Eq. (7) in the special case $M_{n}=M_{1}=0$, let us turn now to the characteristic of solutions of the general equation (6). Note first of all that the slopes of trajectories in the phase plane, as specified by Eq. (7), must be decreased by an amount $M_{n}+\left[M_{1} /\left(1+3 \beta Y^{2}\right)\right]$ if the slope of the trajectories is to be that specified by Eq. (6). In other words, when $M_{n}$ and $M_{1}$ are not zero, damping occurs and the slopes in the phase plane must be adjusted accordingly. Conceivably, then, one would begin with a solution of Eq. (7), sketch in a number of isoclines (i.e., a number of tangent line elements); adjust the slopes and draw new isoclines for Eq. (6), and then sketch in the trajectories for Eq. (6) and have a picture of the performance in the large for the general case.

For the general case, however, it was more convenient to use an analog computer which sketched the trajectories for the following cases: 


$$
\begin{gathered}
\beta=-\frac{4}{300}, \quad r=2.0 \\
M_{1}=0.2, M_{n}=0 \quad \text { in Fig. } 10 \\
M_{1}=1.0, M_{n}=0 \quad \text { in Fig. } 11 \\
M_{1}=0, M_{n}=0.2 \text { in Fig. } 12 \\
M_{1}=0, \quad M_{n}=1.0 \text { in Fig. } 13
\end{gathered}
$$

Note that if $\beta>0$, the quantity $M_{n}+\left[M_{1} /\left(1+3 \beta Y^{2}\right)\right]$ representing the change in slope is always positive and the effect will be the creation of inward spirals toward the value $r$. If $\beta<0$, the change in slope is also positive for bounded solutions since the quantity $\left(1+3 \beta Y^{2}\right)$ is always positive for bounded solutions without damping. Moreover, $\left(1+3 \beta Y^{2}\right)<10$ is not possible in a physical situation.

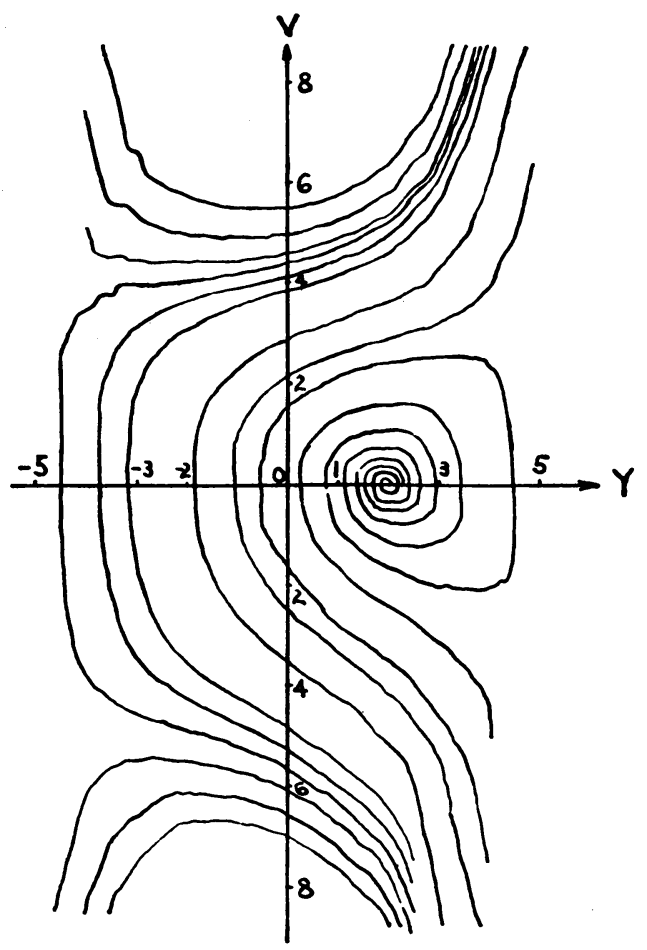

Fra. 10. Computer solution, nonlinear capacity, for $\beta=-4 / 300, r=2.0, M_{1}=0.2$, and $M_{n}=0$.

8. Bounded solution when $M_{n} \neq 0$ and $M_{1}=0$. It can be shown from Figs. 12 and 13 that the higher the value of $M_{n}$, the larger the bounded region. This bounded region for any particular value of $M_{n}$ may be obtained by numerical integration of Eq. (6) provided that the initial values of $Y$ and $V$ are known. It is logical to consider the upper right corner of the bounded region in Fig. 6 as a point through the integral curve. For any given value of $r$ this point is determined by Eq. (23) as shown in the following example, where $r=2, Y_{c}=5.0$, and $V_{c}=2.7387$. 


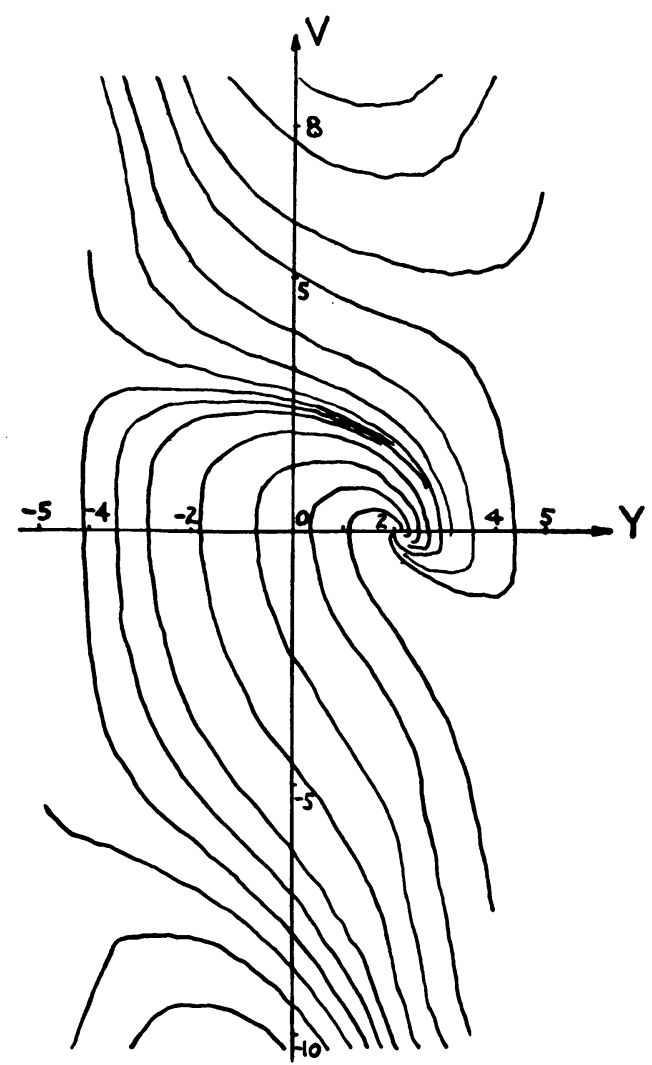

Fra. 11. Computer solution, nonlinear capacity, for $\beta=-4 / 300, r=2.0, M_{1}=1.0$ and $M_{n}=0$.

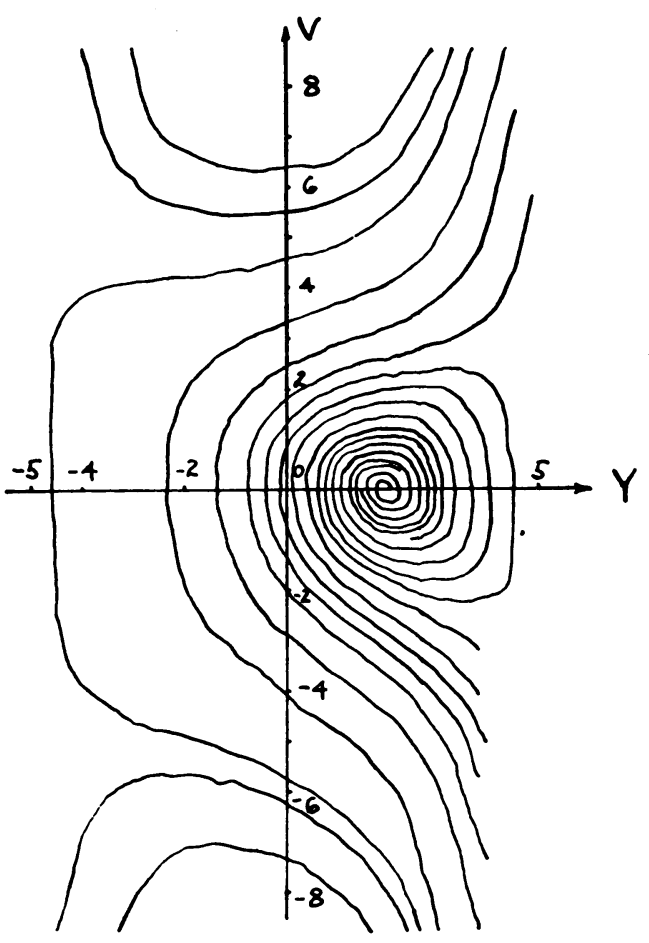

Fig. 12. Computer solution, nonlinear capacity, for $\beta=-4 / 300, r=2.0, M_{1}=0$ and $M_{n}=0.2$. 


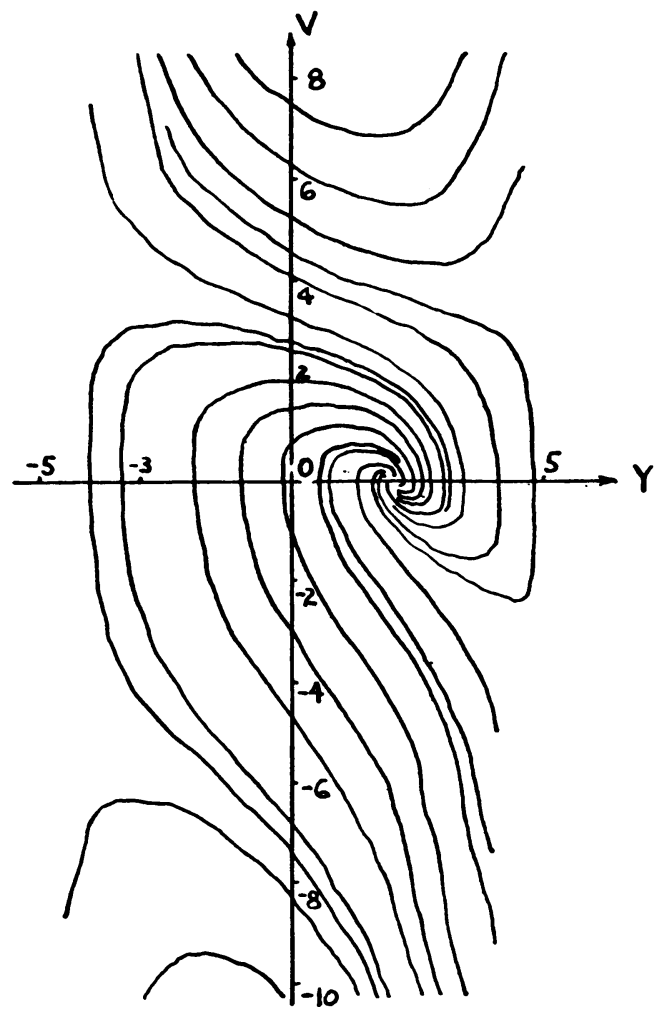

Fra. 13. Computer solution, nonlinear capacity, for $\beta=-4 / 300, r=2.0, M_{1}=0$ and $M_{m}=1.0$.

A closer examination of Fig. 6 and Eq. (23) indicates that this corner point obviously has two distinct slopes - one being infinite, the other being finite, depending upon the direction of approach to the point. To determine the finite slope Eq. (6) is rewritten in the following form:

$$
\lim _{1+3 \beta Y^{3} \rightarrow 0} \frac{d V}{d Y}=-M_{n}+\lim _{1+3 \beta Y^{2} \rightarrow 0} \frac{r-Y\left(1+6 \beta V^{2}\right)}{V\left(1+3 \beta Y^{2}\right)} .
$$

By denoting the limit values of $V$ and $Y$ as $V_{c}$ and $Y_{c}$ respectively, it can be shown that

$$
\left(\frac{d V}{d Y}\right)_{c}=-\left(1+6 \beta V_{c}^{2}\right)\left(18 \beta V_{c} Y_{c}\right)^{-1}-M_{n} / 3 \text {. }
$$

The limiting value of the second derivative of $V$ with respect to $Y$ can also be expressed as

$$
-\left(\frac{d^{2} V}{d Y^{2}}\right)_{c}=V_{c}^{-1}\left(\frac{d V}{d Y}\right)_{c}^{2}+\left(1.25 Y_{c}^{-1}+0.50 M_{n} V^{-1}\right)\left(\frac{d V}{d Y}\right)_{c}+0.25 M_{n} Y_{c}^{-1}
$$

For the same example with $r=2$ and $M_{n}=1.0$, one obtains

$$
\left(\frac{d V}{d Y}\right)_{c}=-.2116, \quad\left(\frac{d^{2} V}{d Y^{2}}\right)_{c}=.0251 \text {. }
$$


To start the integral curve the Taylor's expansion with three terms is adequate.

$$
V\left(Y_{c}+h\right)=V_{c}+h\left(\frac{d V}{d Y}\right)_{c}+\frac{h^{2}}{2}\left(\frac{d^{2} V}{d Y^{2}}\right)_{e}+\cdots
$$

where $h$ is the increment of $Y$. The $m$ th approximation of $f$ may be defined as

$$
\frac{d V}{d Y}=f_{m}(Y)=-M_{n}+\frac{r-Y_{m}\left(1+6 \beta V_{m}^{2}\right)}{V_{m}\left(1+3 \beta Y_{m}^{2}\right)} \text {. }
$$

The above formulae together with the following Simpson's rule constitutes the method of successive approximation in numerical integration.

$$
V_{m}(a+2 h)=V(a)+\frac{h}{3}\left[f(a)+4 f(a+h)+f_{m-1}(a+2 h)\right] .
$$

\begin{tabular}{|c|c|c|c|c|c|}
\hline$Y$ & $\boldsymbol{V}$ & $d V / d Y$ & $Y$ & $\boldsymbol{V}$ & $d V / d Y$ \\
\hline 5.0 & 2.7387 & -.2116 & -5.0 & -4.1833 & -.4130 \\
\hline 4.8 & 2.7815 & -.2166 & -4.8 & -4.2671 & -.4254 \\
\hline 4.6 & 2.8253 & -.2216 & -4.6 & -4.3535 & -.4377 \\
\hline 4.4 & 2.8702 & -.2283 & -4.4 & -4.4425 & -.4542 \\
\hline 4.2 & 2.9165 & -.2336 & -4.2 & -4.5350 & -.4680 \\
\hline 4.0 & 2.9637 & -.2401 & -4.0 & -4.630 & -.484 \\
\hline 3.6 & 3.063 & -.253 & -3.6 & -4.830 & -.519 \\
\hline 3.2 & 3.167 & -.269 & -3.2 & -5.045 & -.558 \\
\hline 2.8 & 3.278 & $-\because 286$ & -2.8 & -5.277 & -.603 \\
\hline 2.4 & 3.396 & -.306 & -2.4 & -5.528 & -.655 \\
\hline 1.6 & 3.662 & -.356 & -1.6 & -6.102 & -.787 \\
\hline 0.8 & 3.972 & -.429 & -0.8 & -6.802 & -.976 \\
\hline 0 & $\begin{array}{l}4.359 \\
\text {. }\end{array}$ & -541 & 0 & -7689 & -1260 \\
\hline 0.8 & 4.857 & -.727 & 0.8 & -8.865 & -1.721 \\
\hline 1.6 & 5.565 & -1.073 & 1.6 & -10.540 & -2.545 \\
\hline 2.4 & 6.677 & 1.809 & & & \\
\hline
\end{tabular}

\section{TABLE 1}

Bounded region with damping term $M_{n}$ for $r=2, M_{n}=1.0, M_{1}=0$

The same technique can be applied to the lower left corner of the bounded region in Fig. 13. The results are listed in Table 1 and plotted in Fig. 14. This is to say that two integral curves determine this bounded region.

On the other hand, for $M_{n}=0.2$ and $M_{1}=0$ there exists only one integral curve. It is sufficient to determine the bounded region by this simple curve alone.

9. The general bounded solutions. The value of $M_{1}\left(1+3 \beta Y^{2}\right)^{-1}$ approaches infinity as $\left(1+3 \beta Y^{2}\right)$ becomes zero. However, the corner point with two distinct slopes shifts to a new position by letting

$$
-Y_{c}\left(1+6 \beta V_{c}^{2}\right)-M_{1} V_{c}+r=0,
$$

from which is obtained

$$
V_{c}=-\frac{M_{1}}{12 \beta Y_{c}} \pm\left[\left(\frac{M_{1}}{12 \beta Y_{c}}\right)^{2}+\frac{r-Y_{c}}{6 \beta Y_{c}}\right]^{1 / 2} .
$$




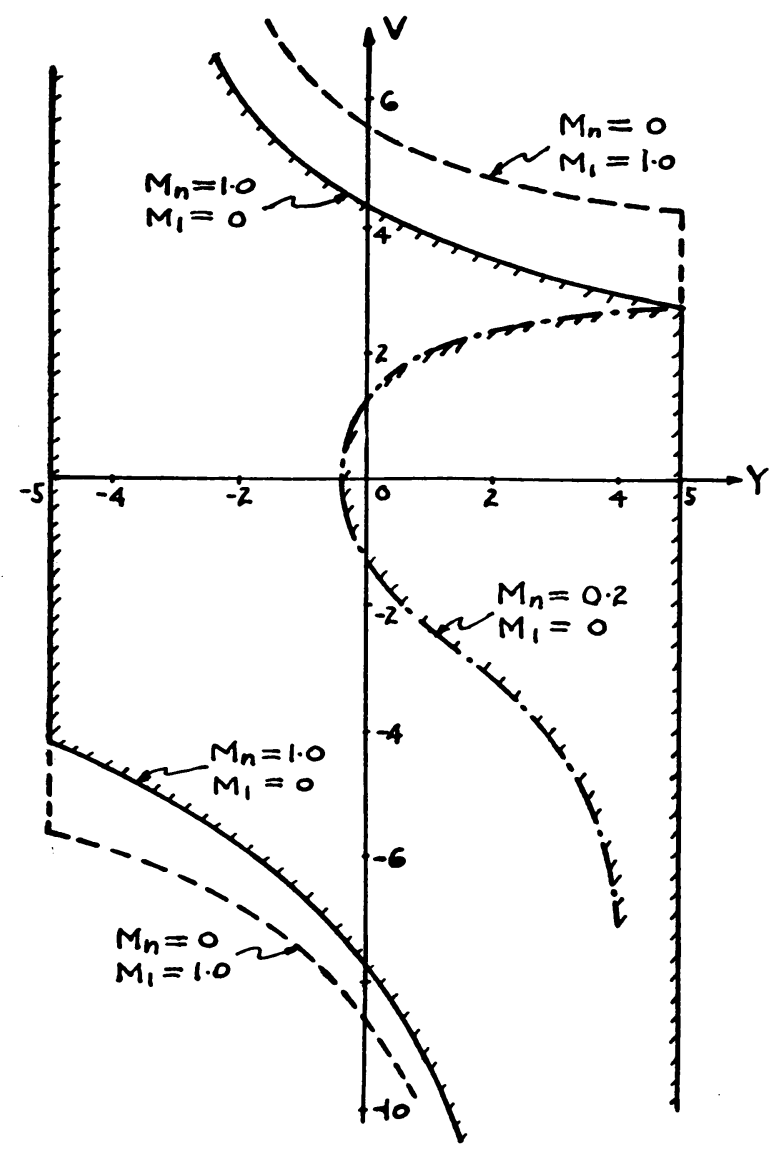

Fra. 14. General bounded regions for $\beta=-4 / 300$ and $r=2.0$.

The limiting value of the finite slope and second derivatives are also obtained as

$$
\begin{gathered}
\left(\frac{d V}{d Y}\right)_{c}=-\left(1+6 \beta V_{c}^{2}+6 \beta V_{c} Y_{c} M_{n}\right)\left(18 \beta V . Y_{c}+M_{1}\right)^{-1} \\
-\left(\frac{d^{2} V}{d Y^{2}}\right)_{c}=\left[V_{c}^{-1}\left(\frac{d V}{d Y}\right)_{c}^{2}+\left(1.25 Y_{c}^{-1}+0.50 M_{n} V^{-1}\right)\left(\frac{d V}{d Y}\right)_{c}+0.25 M_{n} Y_{c}^{-1}\right] \\
\cdot\left[1+M_{1} / 24 \beta Y_{c} V_{c}\right]^{-1} .
\end{gathered}
$$

The same method is used in numerical integration. The results are plotted in Fig. 14 for $r=2, M_{1}=1.0$, and $M_{n}=0$. Figure 14 indicates that the bounded region with $M_{1}=1.0$ and $M_{n}=0$ is bigger than that with $M_{n}=1.0$ and $M_{1}=0$. Thus it can be concluded that the $M_{1}$ term yields more weight in damping than the $M_{n}$ term. For the same type of damping, the lower the value of the $M_{n}$ or the $M_{1}$ term, the smaller the bounded region. This can be shown by comparing Figs. 10 and 11, or Figs. 12 and 13.

For $M_{1}=0$ and $M_{n}$ being finite, there are always some unbounded solutions of the differential equation. This is also true for $M_{n}=0$ and $M_{1}$ being finite, except the value of $V_{c}$ being higher. 
10. Further physical applications. An electric circuit with linear resistance and capacitance in series with a coil having inserted magnetic material can serve as a good example for illustrating more explicitly the results in physical terms. The coil has $N$

(a)

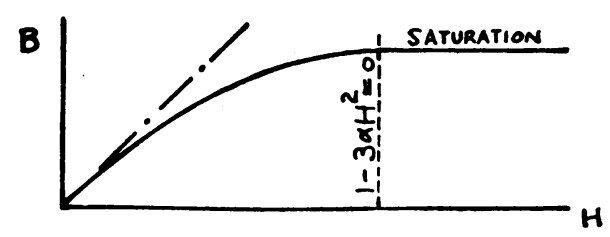

(b)

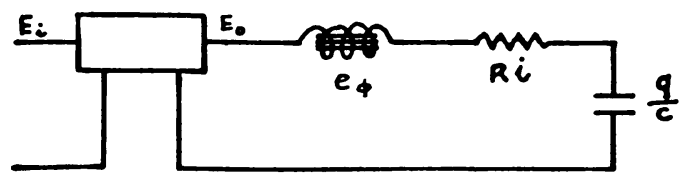

(C)

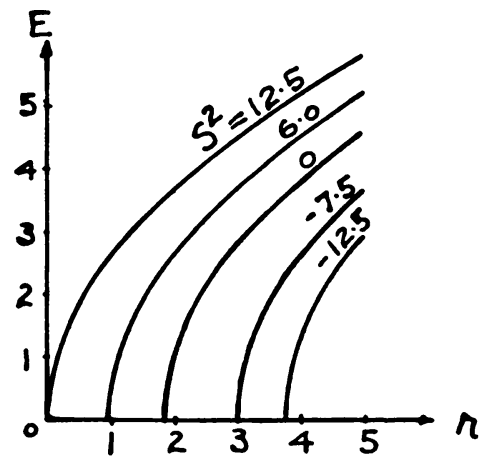

Fro. 15. Nonlinear circuit: a) saturation, b) circuit elements, c) $E$ denotes $V\left(1+3 \beta Y^{2}\right)$ as $1+3 \beta Y^{2} \rightarrow 0$

turns with a magnetic flux $\phi$, thus the emf across the terminals of the coil as shown in Fig. 15(a) is

$$
e_{\phi}=\frac{d}{d t}(N \phi)
$$

The differential equation can be written as

$$
\frac{d(N \phi)}{d t}+R \frac{d q}{d t}+\frac{q}{c}=E_{0}
$$

To solve the above equation it is necessary to determine the relation between $\phi$ and $q$. If the flux density is $B$ and the magnetising force is $H$, the relation between $B$ 
and $H$ before saturation may be approximated by

$$
B=K\left(H-\alpha H^{3}\right),
$$

where

$$
K=\text { constant, } \alpha=\text { nonlinear parameter. }
$$

Let $\mu$ be the incremental permeability of the material, then by differentiating Eq. (43)

$$
\mu=\frac{d B}{d H}=K\left(1-3 \alpha H^{2}\right) .
$$

If $H$ is small, $\mu$ is approximately equal to $K$ as its limiting value. The flux density is saturated at

$$
\frac{d B}{d H}=\mu\left(1-3 \alpha H^{2}\right)=0 .
$$

Thus the $B-H$ relation can be defined only within the region

$$
1-3 \alpha H^{2}>0 \text {, }
$$

or

$$
H^{2}<1 / 3 \alpha .
$$

Substituting the value of $H=N i 1^{-1}$ and $B=\phi A^{-1}$.

$$
\phi=S^{-1} N\left(i-\gamma i^{3}\right),
$$

where $A$ is the cross section area of the material, 1 the length of the magnetic circuit, $i$ the current, $S=1 K^{-1} A^{-1}$ the incremental reluctance, and $\gamma=\alpha N^{2} 1^{-2}$ a nonlinear parameter.

Thus,

$$
e_{\phi}=\frac{d}{d t}(N \phi)=L \frac{d}{d t}\left(i-\gamma i^{3}\right),
$$

where $L=S^{-1} N^{2}=$ inductance for the linear portion.

By substituting $e_{\phi}$ in Eq. (42), the differential equation becomes

$$
L \frac{d}{d t}\left(i-r i^{i^{3}}\right)+R i+\frac{q}{c}=E_{0} .
$$

The differential of the above equation will yield,

$$
L \frac{d^{2}}{d t^{2}}\left(i-r i^{3}\right)+R \frac{d i}{d t}+\frac{i}{c}=\frac{d E_{0}}{d t} .
$$

For a step change of $E_{0} d E_{0} / d t=0$ it can be seen that this is reduced to

$$
\frac{d^{2}}{d \sigma^{2}}\left(i-\gamma i^{-3}\right)+\left(R L^{-1 / 2} C^{1 / 2}\right) \frac{d i}{d \sigma}+i=0,
$$


where

$$
\sigma=(L C .)^{-1 / 2} t,
$$

or

$$
\frac{d^{2}}{d \sigma^{2}}\left(Y+\beta Y^{3}\right)+M_{1} \frac{d Y}{d \sigma}+Y=0,
$$

where $i=I Y, \beta=-\gamma I^{2}=-4 / 300, M_{1}=R L^{-1 / 2} C^{1 / 2}$, and $I$ is a reference current.

For a control device the system sometimes follows a ramp type signal defined by

$$
E_{0}=\tau t \text {, }
$$

where $\tau$ is a constant.

The system will have a differential equation

$$
\frac{d^{2}}{d \sigma^{2}}\left(i-\gamma i^{3}\right)+\left(R L^{-1 / 2} C^{1 / 2}\right) \frac{d i}{d t}+i=\tau
$$

with the starting conditions at $t=0, i=i_{0}, d i / d t=0$. Equation (52) can be expressed in nondimensional form as

$$
\frac{d^{2}}{d \sigma^{2}}\left(Y+\beta Y^{3}\right)+M_{1} \frac{d Y}{d \sigma}+Y=r,
$$

where $r=\tau I^{-1}$ at $\sigma=0, Y=Y_{0}=i_{0} I^{-1}, d Y / d \sigma=0$.

By knowing the values of $L, R, C, \gamma, \tau$ and $i_{0}$ it is possible to compute the parameters $L, M_{1}, r$ and $Y_{0}$ with the fixed value of $\beta=-4 / 300$. To determine whether the solution is bounded it is a simple matter to examine the starting conditions in the phase plane curves for the above nondimensional parameters.

The definition of boundedness as given in previous discussions is entirely mathematical. The unbounded solution was interpreted as the value of $V$ not bounded. However, close examination of Eq. (15) indicates that the product of $V$ and $\left(1+3 \beta Y^{2}\right)$ is bounded. This can be written as

$$
V\left(1+3 \beta Y^{2}\right)=\left[S^{2}-\left(Y^{2}+1.5 \beta Y^{4}\right)+2 r\left(Y+\beta Y^{3}\right)\right]^{1 / 2} .
$$

The curves in Fig. (15) give the values of $V\left(1+3 \beta Y^{2}\right)$ as $\left(1+3 \beta Y^{1}\right)$ approaches zero. It can be proved that the damping terms $M_{1}$ and $M_{n}$ do not affect these values. Equation (6) may be rearranged as

$$
\frac{d}{d \sigma}\left(Y+\beta Y^{3}\right)=V\left(1+3 \beta Y^{2}\right)=\frac{r-Y\left(1+6 \beta V^{2}\right)}{(d V / d Y)+M_{n}+M_{1}\left(1+3 \beta Y^{2}\right)^{-1}} .
$$

As $\left(1+3 \beta Y^{2}\right)$ approaches zero, $V^{2}$ and $d V / d Y$ are of higher order than $\left(1+3 \beta Y^{2}\right)^{-1}$.

The importance of introducing the above analysis is that the voltage $e_{\phi}$ is always finite at the saturation flux. This is because $e_{\phi}$ can be expressed in the form of Eq. (55). Physically the rate of change of $Y$, (i.e., $V$ ), rises very rapidly as $Y$ approaches $(1-1 / 3 \beta)^{1 / 2}$, but the value of $Y$ is only defined within this region.

To carry the physical problem to a new region, the final condition of the nonlinear differential equation is of interest.

$$
\frac{q}{c}=\alpha t_{f}-R i_{f}-e_{s},
$$


where $i_{f}$ and $e_{f}$ are final values of $i$ and $e_{\phi}$ respectively, and $t_{f}$ is the time duration for the current $i$ becoming $i_{j}$. The new region has a horizontal line on the $B$ vs. $H$ curve after the flux is saturated, $\phi$ being constant. It is therefore concluded that the value of $e_{\phi}$ suddenly drops to zero outside the nonlinear region. The system will then become a linear $R C$ circuit with the same ramp input

$$
R \frac{d q}{d t}+\frac{q}{c}=\tau t .
$$

The starting conditions of this linear differential equation are the same as the final conditions of the nonlinear differential equation.

11. Conclusions. The solution of the system of differential equations (3a) and (3b) can be conveniently represented in the $Y V$-phase plane and various sets of graphs made to show the effect of varying the parameters $\beta, r, M_{n}$ and $M_{1}$. For a positive $\beta$ the system is always bounded and the frequency tends to be slower than a corresponding linear case. For a negative $\beta$ with no damping the system is bounded within a horseshoe region and the frequency tends to be faster. If the system is bounded without damping, it is always bounded with damping. The effect will be a spiraling towards the value $r$ in the phase plane.

In a physical system an unbounded solution may be re-defined by extending to a new region beyond its boundary. The analysis can be applied to liquid level controlled systems or circuits with flux saturation. 